\title{
Techniques: Utility of a 3D printed bladder model for teaching minimally invasive urethrovesical anastomosis
}

\author{
Nathan C. Wong, $M D^{\prime}$; Jen Hoogenes, PhD(c)'; Yanbo Guo, $M D^{\prime}$; Mackenize A. Quantz, MD²; \\ Edward D. Matsumoto, $M D^{\prime}$
}

'McMaster University, Hamilton ON; 2Simply Simulators, London, ON; Canada

Cite as: Can Urol Assoc J 2017;11 (7):E321-2. http://dx.doi.org/10.5489/cuai.4262 Published online July 11, 2017

\section{Introduction}

The urethrovesical anastomosis (UVA) is one of the most challenging steps during a minimally invasive radical prostatectomy. Not surprisingly, minimally invasive, in particular laparoscopic, prostatectomy is associated with a steep learning curve. ${ }^{1}$ With competency-based training on the horizon, as well as the recent reduction in resident work hours, surgical educators have shifted some training outside of the operating room into surgical skills labs. To reduce learning curves and improve resident education at our centre, we use a hands-on 3D printed bladder bench model to emulate the UVA task during a minimally invasive prostatectomy.

\section{Evolution of the 3D printed bladder model}

Early versions of the bladder model were fashioned from latex and subsequently silicone. In two randomized controlled studies, these models were shown to be effective teaching tools. Training on a task-specific bench platform using the latex bladder model was superior to basic laparoscopic suturing drills on a foam pad for performing a laparoscopic UVA on the bench model, ${ }^{2}$ as well as performing a laparoscopic UVA on an live anesthetized pig. ${ }^{3}$ It was concluded from these studies that skills learned on the latex bladder model transfer well to both a low-fidelity bench setting and a high-fidelity live animal.

Following this, we aimed to further improve characteristics of the model. A new, low-cost 3D printed bladder model (Simply Simulators, London, ON, Canada) is currently used. The conceptual simulator was developed using computer-aided design software and rapid prototyping was performed using a Lulzbot Taz 4 3D printer (Loveland, CO, U.S.). After several iterations and prototyping, the final shape of the model has anatomical characteristics (e.g., size and thickness) more similar to that of the human bladder and urethra than our previous versions. Final simulator models are produced using a unique polymer with characteristics similar to the human bladder with regards to handling, incising, cutting, needle penetration, and suture pull-through.

We have recently used this $3 \mathrm{D}$ printed bladder model to investigate robotic skills transfer from two virtual reality robotic simulators, the dV-Trainer (Mimic Technologies, Inc., Seattle, WA, U.S.) and the da Vinci Surgical Skills Simulator, to performing the UVA task on the bladder model using the da Vinci Surgical System (Intuitive Surgical, Sunnyvale, CA, U.S.). ${ }^{4}$ In post-simulation surveys, participants with previous robotic-assisted laparoscopic prostatectomy experience reported that they felt the $3 \mathrm{D}$ bladder model was both realistic and useful in simulating the UVA task.

\section{Performing a UVA task on the 3D printed bladder model}

\section{Setup and positioning}

To simulate the setup of a minimally invasive prostatectomy, the $3 \mathrm{D}$ printed bladder model is fixated within a realistic pelvic silicone torso (Limbs \& Things, Bristol, U.K.) (Fig. 1). Three ports are placed through the silicone torso, one at the umbilicus for the endoscopic camera and two working ports placed laterally, one on each side, to facilitate intracorporeal suturing. This system allows trainees to practice essential steps of a laparoscopic or robotic prostatectomy in a safe and risk-free environment outside of the operating room. Not only do trainees have the opportunity to deliberately practice proper needle control and suture handling, they also learn how to operate within the strict confines of the narrow pelvis.

\section{Urethrovesical anastomosis}

To begin the task, the trainee uses Metzenbaum scissors to transect the bladder model at the urethrovesical junc- 


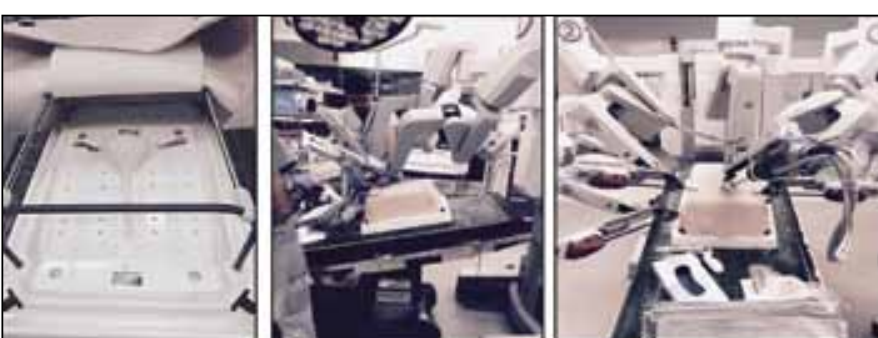

Fig. 1. Setup of $3 \mathrm{D}$ printed bladder model within the realistic pelvic silicone torso.

tion. Two $15 \mathrm{~cm}$ 3-0 Monocryl ${ }^{\circledR}$ sutures (one dyed and one not dyed) tied tail to tail with an RB needle at each end is introduced into the pelvis from one of the working ports. For orientation and communication purposes, while looking into the pelvis from the head in a supine position, the 12 $\mathrm{o}^{\prime}$ clock position represents the anterior urethra/bladder and the 6 o'clock position represents the posterior urethra/bladder. Needle drivers are used to place the sutures out-to-in at the $6 \mathrm{o}^{\prime}$ clock bladder position using each arm of the suture. The sutures are then placed in-to-out at the 6 o'clock urethral position. The running anastomosis is performed along each side connecting the bladder to the urethra from the 6-9 o'clock on the left side and the 6-3 o'clock on the right side (Fig. 2). An 18 French Foley catheter is introduced from the urethral end of the model beyond the completed posterior half of the anastomosis. The anastomosis is then completed over the anterior portion and both ends meet at the $12 \mathrm{o}^{\prime}$ clock urethral position. The needles are cut off and removed from the pelvis. The sutures are then tied together with three square knots to complete the UVA task.

\section{Assessment and feedback}

After completion of the task, the bladder model can be taken out of the torso and the final end-product can be visually inspected and assessed (Fig. 3). The endoscopic camera can record performances that can later be reviewed by the trainee, as well as surgical educators to obtain valuable feedback. Three assessment instruments used in our previous publications can objectively evaluate trainees' performances: 1 ) a global rating scale; ${ }^{2} 2$ ) a procedure-specific end-product checklist; ${ }^{3}$ and 3 ) a procedure-specific checklist. ${ }^{3}$
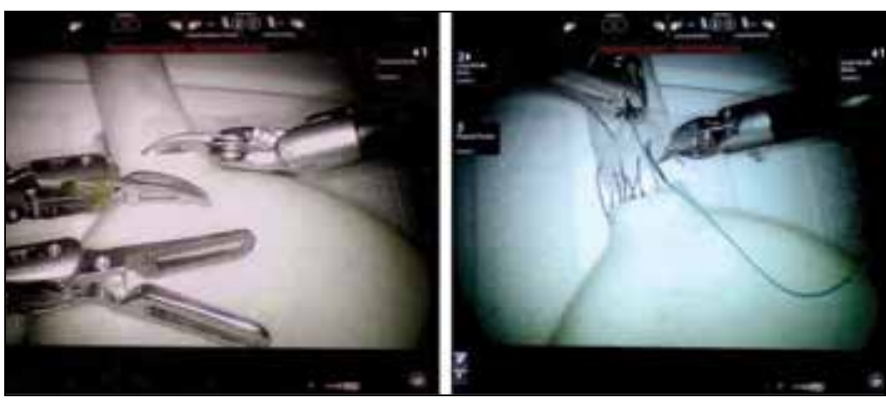

Fig. 2. Urethrovesical anastomosis on 3D printed bladder model.
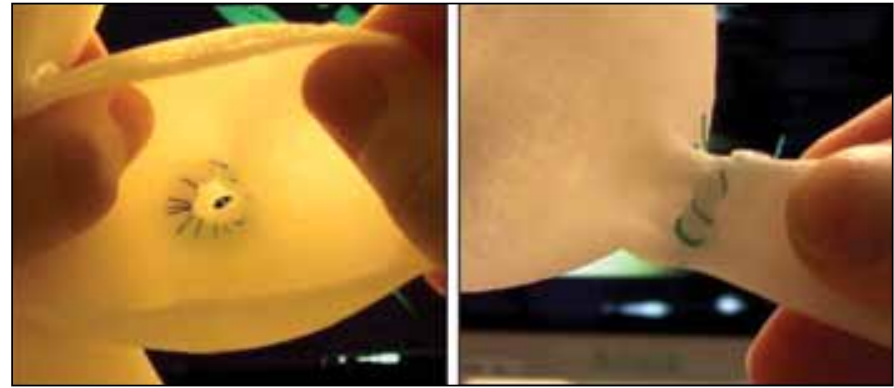

Fig. 3. Final end product of urethrovesical anastomosis.

\section{Conclusion}

At our centre, surgical residents have access to the Centre for Minimal Access Surgery (CMAS), which provides stateof-the-art facilities, education, and training for minimally invasive procedures. Here, residents may use the 3D printed bladder models to practice the UVA task and continue to refine their surgical skills outside of the operating room in a safe and effective manner.

Competing interests: Dr. Quantz is the founder of Simply Simulators. The remaining authors report no competing personal or financial interests.

This paper has been peer-reviewed.

\section{References}

1. Vickers AJ, Savage CJ, Hruza $M$, et al. The surgical learning curve for laparoscopic radical prostatectomy: A retrospective cohort study. Lancet Oncol 2009;10:475-80. https://doi.org/10.1016/S14702045(09)70079-8

2. Sabbagh R, Chatterjee $S$, Chawla A, et al. Task-specific bench model training vs. basic laparoscopic skills training for laparoscopic radical prostatectomy: A randomized, controlled study. Can Urol Assoc J 2009;3:22-30. https://doi.org/10.5489/cuaj.1011

3. Sabbagh R, Chatterjee S, Chawla A, et al. Transfer of laparoscopic radical prostatectomy skills from bench model to animal model: A prospective, single-blind, randomized, controlled study. J Urol 2012;187:18616. https://doi.org/10.1016/i.uro.2011.12.050

4. Wong NC, Hoogenes J, Alharbi B, et al. Robotic surgical skills acquisition in trainees: A randomized comparison of the two robotic trainers and trainees' skills transfer to a 3D printed simulated surgical task in the operating room. J Urol 2016;195:E215-6.

Correspondence: Dr. Nathan C. Wong, McMaster University, Hamilton, ON, Canada; nathan.wong@medportal.ca 